\section{Tuberkulose: Kurze Chemoprophylaxe schützt HIV-Patienten}

Swindells S et al. One Month of Rifapentine plus Isoniazid to Prevent HIV-Related Tuberculosis. N Engl J Med 2019; doi:10.1056/NEJMoa1806808

Die Tuberkulose stellt eine häufige Todesursache von HIV-Patienten dar, Präventionsstrategien für Menschen in Hochrisikogebieten werden händeringend gesucht. Isoniazid über 9 Monate ist zwar effektiv, geht aber mit teils schweren Nebenwirkungen und schlechter Compliance einher. Swindells und Team haben daher nun eine Nicht-Unterlegenheitsstudie (Phase III) durchgeführt und Rifapentin plus Isoniazid für 1 Monat mit dem Standard verglichen.

Eine der Haupttodesursachen von Patienten mit HIV-Infektion ist die Tuberkulose. Aus diesem Grund hat sich insbesondere in Hochrisikogebieten die Chemoprophylaxe mit Isoniazid über 9 Monate bewährt. Trotz der guten Wirksamkeit bringt diese Strategie allerdings auch Nachteile mit sich. So leiden viele Patienten unter schweren Nebenwirkungen und setzen ihre Medikamente ab.

Nachdem sich die Kombination aus Rifapentin und Isoniazid über nur ! Monat in präklinischen Studien an Mäusen als sicher und wirksam erwiesen hat, haben Swindells und sein Team nun eine entsprechende Phase-III-Studie durchgeführt. In dieser randomisierten und offenen Nicht-Unterlegenheitsstudie wollten sie herausfinden, ob die oben genannte Kombinationstherapie dem Standard mit Isoniazid über 9 Monate nicht unterlegen ist. Die Untersuchung fand zwischen Mai 2012 und November 2014 an 45 Zentren in 10 Ländern mit Gebieten mit hohem Tuberkuloserisiko statt.

Geeignete Patienten erfüllten folgende Kriterien:

- Alter ab 13 Jahren,

- bestätigte HIV-Infektion,

- wohnhaft in einer Region mit hohem Tuberkuloserisiko oder
- positives Testergebnis für eine latente Tuberkulose.

Die wichtigsten Ausschlusskriterien waren eine bekannte oder vermutete aktive Tuberkulose, eine laufende Tuberkulosetherapie, Schwangerschaft oder Stillzeit sowie Lebererkrankungen.

Die Studienautoren bildeten 2 Gruppen:

- Gruppe 1: Kombinationstherapie aus Rifapentin und $300 \mathrm{mg}$ Isoniazid 1-mal täglich

- Gruppe 2: Monotherapie mit $300 \mathrm{mg}$ Isoniazid über 9 Monate

Die Dosis von Rifapentin in Gruppe 1 wurde nach dem Körpergewicht angepasst, der Zeitraum der Nachbeobachtung erstreckte sich bis November 2017. Als primären klinischen Endpunkt definierten die Autoren die Zeit bis zur ersten Diagnose einer aktiven Tuberkuloseinfektion sowie den durch Tuberkulose bedingten Tod oder den Tod unklarer Ursache. Sekundäre klinische Endpunkte waren Sicherheit, Nebenwirkungsprofil, Tod jeglicher Ursache und Todesfälle ohne Zusammenhang mit einer Tuberkuloseinfektion.

\section{Kombitherapie nicht unterlegen}

3696 Patienten erfüllten die Einschlusskriterien, 3000 von ihnen wurden randomisiert, und die Daten von 2986 von ihnen gingen schließlich in die finale Auswertung mit ein. 54\% aller Teilnehmer waren weiblich, der Median der CD4-positiven Zellen lag bei 470 Zellen pro Kubikmeter mit einem Interquartilabstand von 346-635. 50\% aller Patienten erhielten eine antiretrovirale Therapie, bei $77 \%$ war die Viruslast unbekannt. 97\% aller Studienpatienten lebten in Hochrisikogebieten für Tuberkulose mit über 60 Fällen auf 100000 Einwohner, 692 (23\%) zeigten im Tuberkulin-Hauttest ein positives Ergebnis.

97\% der Patienten in Gruppe 1 mit Kombinationstherapie, aber nur $90 \%$ in Gruppe 2 beendeten die Behandlung. Dieser Unterschied war dabei hochsignifikant. Der primäre Endpunkt wurde bei
32 von 1488 Patienten in Gruppe 1 (2\%) und bei 33 von 1498 Patienten in Gruppe 2 (2\%) festgestellt. Die Inzidenzraten für Tuberkulose pro 100 Personenjahre konten auf 0,65 bei Gruppe 1 und 0,67 bei Gruppe 2 beziffert werden.

Die Forscher stellten bei $6 \%$ aller Patienten aus Gruppe 1 und bei $7 \%$ aller Patienten aus Gruppe 2 ernsthafte Nebenwirkungen fest. In ihrem Diskussionsteil betrachten die Autoren auf Grundlage ihrer Ergebnisse die Nicht-Unterlegenheit der deutlich kürzeren Kombinationstherapie als bestätigt und sehen darin eine vielversprechende Alternative zur Prävention von Tuberkulose von HIV-Patienten in Hochrisikogebieten.

\section{FAZIT}

In dieser Nicht-Unterlegenheitsstudie (Phase III) war die Kombinationstherapie mit Rifapentin und Isoniazid über 1 Monat dem heutigen Standard von 9 Monaten Isoniazid allein zur Chemoprophylaxe bei HIV-Patienten in Tuberkulosegebieten nicht unterlegen. Da Patienten mit Kombinationstherapie zudem seltener an Tuberkulose erkrankten und über weniger Nebenwirkungen berichteten, halten die Autorinnen/Autoren dieses Schema für eine vielversprechende Alternative zur bisherigen Monotherapie.

Dipl.-Psych. Annika Simon, Hannover 